Forum 2016 · 31:337

DOI 10.1007/s12312-016-0100-2

Online publiziert: 13. Juli 2016

○) Springer-Verlag Berlin Heidelberg 2016
Ausschuss Öffentlichkeitsarbeit der Landeskrebsgesellschaften e.V.

\section{E-Zigaretten keinesfalls harmlos}

\author{
Helfen E-Zigaretten beim Rauchstopp oder verführen sie \\ sogar mehr Menschen zum Rauchen?
}

Anlässlich des Weltnichtrauchertags am 31. Mai klärten die Landeskrebsgesellschaften in ihren Bundesländern über die Wirkung von E-Zigaretten und E-Shishas auf.

Das Rauchen von Zigaretten ist erwiesenermaßen gesundheitsschädlich und kann zu Lungenkrebs führen. Mehr als 90 \% der Männer, die an dem Krebsleiden erkranken, sind oder waren Raucher. Bei Frauen gehen etwa $65 \%$ der Lungentumore auf das Rauchen zurück. Doch wie sieht es mit der Gefährdung durch E-Zigaretten und E-Shishas aus? Die Datenlage hier ist nicht so eindeutig wie beim konventionellen Tabakkonsum.

Um dennoch etwas Klarheit für den Verbraucher zu schaffen, haben die Landeskrebsgesellschaften beim Deutschen Krebsforschungszentrum (DKFZ) in Heidelberg nachgefragt. Die dort ansässige „Stabsstelle Krebsprävention“ und das „WHO-Kollaborationszentrum für Tabakkontrolle“ werten kontinuierlich die aktuelle Studienlage aus. Dr. Katrin Schaller vom DKFZ fasst den gegenwärtigen Wissensstand so zusammen: „Sicherlich sind E-Zigaretten weniger schädlich als normale Zigaretten, aber harmlos sind sie keinesfalls.

\section{Elektronische Zigaretten produzieren gefährliche Dämpfe}

So erzeugen E-Zigaretten und E-Shishas inhalierbare Aerosole, die eine Reihe von entzündungsfördernden, sensibilisierenden und Gen-toxischen Substanzen enthalten. In dem chemischen Gemisch befinden sich unter anderem Propylenglykol, Formaldehyd, Acetaldehyd und nicht zuletzt Nikotin - Schadstoffe, die als überaus bedenklich und zum Teil als krebserregend gelten. „Gesund ist das alles nicht" so Schaller, wenn auch das Gefährdungspotenzial von Produkt zu Produkt variiere. Studien zeigen, dass die Zusammensetzung der Chemikalien, die Gerätetechnik und hier insbesondere die Verdampfertemperaturen sowie das Nutzerverhalten maßgeblich das Gesundheitsrisiko bestimmen. Als Faustregel gilt: Je höher die Betriebstemperatur und je tiefer inhaliert wird, desto schädlicher für die Lunge. $\mathrm{Da}$ rüber hinaus kann die gesundheitliche Belastung Dritter nicht ausgeschlossen werden, da auch beim Konsum elektrischer Zigaretten Nikotin und krebserregende Substanzen in die Raumluft abgegeben werden.

\section{Unklare Studienlage zu Rauchausstieg und -einstieg}

E-Zigaretten werden vorwiegend von Rauchern und denjenigen, die einen Rauchausstieg erwägen, genutzt. Inwieweit E-Zigaretten beim Rauchausstieg wirklich helfen können, ist bislang aber nicht abschließend geklärt. Die systematischen Übersichtsarbeiten zu diesem Thema kommen $\mathrm{zu}$ widersprüchlichen Ergebnissen. Jedoch lauten die Schlussfolgerungen übereinstimmend, dass aufgrund der geringen Evidenz eine eindeutige Empfehlung zum jetzigen Zeitpunkt nicht möglich ist. Darum wird in der S3 Leitlinie zur Behandlung des abhängigen Tabakkonsums derzeit die E-Zigarette nicht zur Suchtentwöhnung empfohlen. Ähnlich schwach und uneinheitlich ist die Studienlage hinsichtlich des Raucheinstiegs. Auch hier gibt es bislang noch keine gesicherte Evidenz, ob die elektronische Rauchware mehr junge Menschen zum Rauchen verführt.

Dennoch bleibt zu befürchten, dass E-Zigaretten und E-Shishas die Reiz- schwelle für Jugendliche senken, da die E-Inhalationsprodukte vielfach als gesündere Alternativen zum Rauchen beworben werden und obendrein noch mit Aromen nach Erdbeere oder Schokolade locken. Die Gefahr dabei: E-Zigaretten enthalten - neben anderen gesundheitsschädlichen Substanzen - eben auch den Suchtstoff Nikotin, der physisch und psychisch abhängig machen kann. Nicht zuletzt deshalb hat der Gesetzgeber zum 1. April 2016 den Verkauf von E-Zigaretten und E-Shishas an Kinder und Jugendliche verboten.

\section{$70 \%$ betreiben einen dualen Konsum}

„In beiden Fragestellungen sind also derzeit keine klaren Aussagen möglich“ betont Präventionsexpertin Dr. Katrin Schaller. In puncto Rauchstopp weist die Expertin aber auf einen Umstand hin, der hellhörig macht: $70 \%$ der E-Zigaretten-Nutzer betreiben einen dualen Konsum - kommen also nicht wirklich los von der Zigarette. Sinnvoll sei das Nebeneinander nicht, meint Schaller. „Gesundheitlich bringt das nicht viel - dafür ist ein vollständiger Rauchausstieg nötig“, so die Expertin. „Nach aktuellem Wissensstand können wir die E-Zigarette nicht zum Rauchstopp empfehlen.“

\section{Kontakt}

Ausschuss für Öffentlichkeitsarbeit der

Landeskrebsgesellschaften

Sprecherin: Gabriele Brückner

Bayerische Krebsgesellschaft e.V.

Tel: 0895488400

brueckner@bayerische-krebsgesellschaft.de 